\title{
Neutron Capture and Transmission Measurements and Resonance Parameter Analysis of Niobium
}

\author{
NJ Drindak, JA Burke, G Leinweber, JA Helm, JG Hoole, \\ RC Block, Y Danon, RE Slovacek, BE Moretti, CJ Werner, \\ ME Overberg, SA Kolda, MJ Trbovich, DP Barry
}

This report was prepared as an account of work sponsored by the United States Government. Neither the United States, nor the United States Department of Energy, nor any of their employees, nor any of their contractors, subcontractors, or their employees, makes any warranty, express or implied, or assumes any legal liability or responsibility for the accuracy, completeness or usefulness of any information, apparatus, product or process disclosed, or represents that its use would not infringe privately owned rights. 


\title{
Neutron Capture and Transmission Measurements And Resonance Parameter Analysis Of Niobium
}

\author{
NJ Drindak, JA Burke, G Leinweber, JA Helm, JG Hoole \\ Lockheed Martin Corporation \\ RC Block, Y Danon, RE Slovacek, BE Moretti, CJ Werner, \\ ME Overberg, SA Kolda, MJ Trbovich, DP Barry \\ Rensselaer Polytechnic Institute (RPI)
}

July 2004

\begin{abstract}
Epithermal neutron capture and transmission measurements were performed using the timeof-flight method at the RPI linac using metallic Nb samples. The capture measurements were made at the 25-meter flight station with a 16-section sodium iodide multiplicity detector and the transmission measurements at the 25-meter flight station with a Li-6 glass scintillation detector. Resonance parameters were determined for all resonances up to $500 \mathrm{eV}$ with a combined analysis of capture and transmission data using the multi-level R-matrix Bayesian code SAMMY. The present results are compared to those presented in ENDF/B-VI, updated through Release 3.
\end{abstract}

1) Introduction

Years ago, the accuracy of measured neutron cross sections exceeded the accuracy of computer codes used for reactor calculations. As advances were made in Monte Carlo methods and as computer power increased, the situation changed. The accuracy of the cross section data is now the limiting factor in many reactor calculations.

The KAPL-RPI Cross Section Group is working to improve the accuracy of measured data. New linac targets have been designed to optimize neutron production in various energy ranges ${ }^{1}$. New neutron detectors have been produced to improve both capture and transmission measurements. A high-efficiency 16-segment multiplicity detector has been developed for capture measurements ${ }^{2}$. An improved transmission detector, with the photomultiplier tubes positioned out of the neutron beam, has been placed in service ${ }^{3}$. Both the capture and transmission detectors use computer controlled sample changers to automatically cycle through sets of samples.

The SAMMY ${ }^{4}$ computer code is being used to analyze the measured data. A special module has been added to the code to model the RPI resolution function. A great deal of effort has been devoted to fitting the parameters for this function. 


\section{2) Experimental Conditions}

\section{A. General}

The RPI linac is used to produce neutrons. A pulsed electron beam (approximately 58 $\mathrm{MeV}$ ) strikes a water-cooled tantalum target. Electrons colliding with the tantalum generate bremsstrahlung gamma rays that in turn produce photoneutrons. The neutrons are moderated and collimated to form a neutron beam. The capture and transmission detectors are located on separate 25-meter flight paths.

Capture and transmission measurements were made at epithermal energies. An effort was devoted to making thermal measurements. However, the extremely low thermal capture cross section of niobium (1.15 barns) prevented a thermal measurement with acceptable accuracy.

The KAPL-RPI data that was analyzed consisted of measurements of six different niobium metal samples. The purity of all the samples was $99.9 \%$. Since natural niobium is a monotope, all of the niobium was ${ }^{93} \mathrm{Nb}$. The principal impurity, tantalum, did not have a significant effect in the energy range that was analyzed. The samples were mounted in aluminum cans for measurement. The effect of the cans was measured by including empty cans in the capture measurements. Table I shows the sample characteristics.

\begin{tabular}{|c|c|c|c|c|}
\hline \multicolumn{5}{|c|}{ Niobium Sample Characteristics } \\
\hline $\begin{array}{c}\text { Nominal } \\
\text { Thickness } \\
\text { (in) }\end{array}$ & $\begin{array}{c}\text { Nominal } \\
\text { Diameter } \\
\text { (in) }\end{array}$ & Measurement & $\begin{array}{c}\text { Number Density } \\
\text { (atom/barn) }\end{array}$ & $\begin{array}{c}\text { Tantalum } \\
\text { Impurity* } \\
\text { (ppm) }\end{array}$ \\
\hline 0.002 & 1.985 & Capture & 0.0002826 & Ta-780 \\
\hline 0.005 & 1.985 & Capture & 0.0007417 & Ta-570 \\
\hline 0.010 & 1.985 & Capture & 0.001192 & Ta-570 \\
\hline 0.020 & 1.985 & Capture & 0.002585 & Ta-570 \\
\hline 0.135 & 1.985 & Transmission & 0.01944 & Ta-570 \\
\hline 0.267 & 1.985 & Transmission & 0.03837 & Ta-570 \\
\hline
\end{tabular}

* All other impurities were an order of magnitude smaller.

All measurements were made using the RPI bounce target with an electron burst width of 40 nsec (neutron burst width is approximately the same). The pulse repetition rate was 200 pulses/sec for capture and 250 pulses/sec for transmission. For transmission, the channel width was 0.125 usec for the lower energy resonances. Above $243 \mathrm{eV}$ the channel width was 0.03125 
$\mu$ sec. For capture, the channel width was $0.25 \mu$ sec for the lower energy resonances. Above 106 $\mathrm{eV}$ the channel width was $0.0625 \mu \mathrm{sec}$.

The time-of-flight (TOF) method is used to measure neutron energy. The interval between the linac burst and the detection of an event defines the flight time of the neutron. Since the flight path length is known precisely, the energy of the neutron can be determined. Using the capture detector as an example, the physically measured flight path from the moderator face to the face of the sample, with one mean free path in the moderator $(0.0055 \mathrm{~m})$ added is $25.566 \mathrm{~m}$. This measurement was confirmed with a time-of-flight measurement of ${ }^{238} \mathrm{U}$, deriving flight path length from precisely known resonance energies. The TOF analyzer used in these experiments was designed and constructed in-house. ${ }^{5,6}$ It uses a $32 \mathrm{MHz}$ crystal-controlled oscillator coupled to a 22-bit scaler. The analyzer operates as a single-start/multiple-stop device. Each linac pulse starts a countdown cycle during which a number of detected neutron events can be recorded. The dead time of the signal counting electronics was estimated to be $1.125 \mu$ sec for capture and $0.6 \mu$ sec for transmission.

A Hewlett-Packard HP-1000 computer controls the data-taking process. During the experiment, data are transferred from the TOF analyzer to the computer memory with Direct Memory Access (DMA). The data-taking software is menu driven. It controls the sample changers, sorts the data into individual spectra, and displays the data as it is accumulated.

\section{B. Capture Detector}

Neutron capture measurements were made with a 16-section multiplicity detector., ${ }^{5,6}$ Each of the $16 \mathrm{NaI}(\mathrm{Tl})$ segments is a gamma scintillation detector with its own RCA 8575 photomultiplier tube. When assembled, the segments form a 12-inch-by-12-inch right circular cylinder with a 3.5-inch through hole along its axis. The cylinder is split normal to its axis into two rings, each hermetically sealed in an aluminum can. Each ring is divided into 8 pie shaped segments, which are light-isolated from each other. The total volume of $\mathrm{NaI}(\mathrm{Tl})$ is 20 liters. The detector is located on the East flight tube 25.566 meters from the bounce target. Flight path length was calibrated with measurements of precisely known uranium resonances.

A computer controlled sample changer was used to precisely locate two-inch diameter metal samples at the center of the capture detector. The sample changer mounts up to eight samples and moves them into the beam one at a time. The capture detector is shielded from scattered neutrons by a boron carbide liner in the bore of the detector that absorbs neutrons scattered from the sample being measured. The boron in the liner is enriched in ${ }^{10} \mathrm{~B}$ to 99.4 weight percent. A six-inch thick, 16,000-pound lead shield surrounding the detector reduces gamma ray background.

\section{Transmission Detector}


Total cross section measurements were made with a ${ }^{6} \mathrm{Li}$ glass scintillation detector. The ${ }^{6} \mathrm{Li}$ glass is 5-inches in diameter and is 0.5 -inch thick. The detector is coupled to a 5-inch diameter photomultiplier tube in line with the neutron flight path. The detector is located on the center flight tube 25.585 meters from the bounce target. Flight path length was calibrated with measurements of precisely known ${ }^{238} \mathrm{U}$ resonances. Samples are precisely located in the beam by an 8-position, computer-controlled sample changer.

\section{Data Reduction}

\section{A. Capture Data}

A neutron capture event in the sample typically gives off several gamma rays that are absorbed in the sodium iodide segments. The energy absorbed in the 16 segments is summed. If the total energy exceeds $1 \mathrm{MeV}$, the event is counted and a 300-nsec timing window is opened. The number of segments with energy greater than $100 \mathrm{keV}$ during this window is recorded as the multiplicity of the event. Multiplicity information is useful in analyzing background and spin states. The multiplicity and the TOF for the neutron causing the event are recorded by the TOF analyzer.

The average linac current during capture runs was about $32 \mu \mathrm{A}$. The duration of an individual data run (meaning one cycle through the set of samples) was about one hour. This time is chosen to minimize data loss in the event of an equipment malfunction, and to average beam intensity variations. Fifty-two capture data runs were performed for this experiment.

After all the data runs were accumulated, a statistical check was done comparing all the data sets for consistency. Data sets were eliminated if the run-to-run variation of the ratio of detector counts to neutron beam monitor counts was more than three standard deviations. (Examination of conditions for these data runs usually indicates machine variations caused by warm up or linac trips). After correcting for dead time, data sets with acceptable statistics were summed in one 'grand sum' data set. Next, the data were normalized to the neutron beam fluence using the neutron beam monitors. For capture data background is measured with an empty sample can holder mounted on the sample changer. The measured background spectra are subtracted from the summed and normalized capture spectra. Finally, the 16 individual multiplicity spectra were collapsed into a single multiplicity-independent spectrum.

$\boldsymbol{Y}_{\boldsymbol{i}}$, the capture yield TOF channel $\boldsymbol{i}$, is given by:

$$
Y_{i}=\frac{C_{i}-B_{i}}{K \phi_{i}}
$$


where $\boldsymbol{C}_{\boldsymbol{i}}$ is the dead-time corrected and monitor-normalized counting rate of the sample measurement,

$\boldsymbol{B}_{\boldsymbol{i}}$ is the background counting rate,

$\boldsymbol{K}$ is the flux normalization factor, and

$\boldsymbol{\Phi}_{i}$ is the unnormalized neutron flux

To determine capture yield the neutron flux spectrum must be determined. The flux spectrum is measured in a separate set of runs. The shape of the neutron flux is determined by placing a black (to neutrons) sample in the capture detector. In this case a ${ }^{10} \mathrm{~B}_{4} \mathrm{C}$ sample, 0.1 inches thick, was used. With a total energy window set to record the $478 \mathrm{keV}$ gamma rays from

neutron absorption in ${ }^{10} \mathrm{~B}$, the energy profile of the neutron beam was measured. This measurement gives the shape of the neutron flux but not its magnitude. The flux profile is usually normalized to the same fluence as the capture data using a black, highly absorbing resonance in the capture data. However, this was not possible in this instance because there were no black resonances in the niobium capture data. Instead, SAMMY was used to normalize the capture data. First SAMMY was used to fit the transmission data and obtain resonance parameters. Then these resonance parameters were used with the capture data to fit capture normalization. The $119 \mathrm{eV}$ resonance was used for normalization because it was a strong capture $(\approx 97 \%)$ resonance and had good statistics. Also, as an s-wave resonance it gave a good normalization to the major resonances, which are all s-waves.

\section{B. Transmission Data}

The transmission data differ from the capture data in that only one time-of flight spectrum is recorded by the time-of-flight analyzer (instead of the 16 spectra collected for the capture detector). The average linac current during transmission runs was about $34 \mu \mathrm{A}$. As with capture, the duration of an individual data run (meaning one cycle through the set of samples) was about one hour for the reasons previously discussed. Eighty-three transmission data runs were performed for this experiment. After all the data runs were accumulated, a statistical check was done comparing all the data sets with each other. Data sets were eliminated if detector counts and neutron beam monitor counts were outside the range of normal statistical fluctuations. The data were then corrected for dead time, and the runs were normalized and summed.

The one notch/two notch method ${ }^{7}$ was used to determine the time dependent background for transmission. This method uses two sets of filters. The first (one notch) set has several different materials, each with a thickness chosen to produce a black notch in the neutron spectrum. The second (two-notch) set is the same as the first set, except with the thickness doubled. Background at the energy of each notch is determined by extrapolating the two-notch background and the one-notch background to obtain the no notch background. The no-notch background points were fitted with a smooth analytical function in TOF for each sample. The resulting expression was used for background correction. 
$\boldsymbol{T}_{\boldsymbol{i}}$, the transmission in TOF channel $i$, was calculated by:

$$
T_{i}=\frac{\left(C_{i}^{S}-K_{S} B_{i}-B_{S}\right)}{\left(C_{i}^{o}-K_{o} B_{i}-B_{o}\right)}
$$

where $C_{i}^{S}$ and $C_{i}^{o}$ are the dead-time corrected and normalized counting rates of the sample and open measurements, respectively,

$\boldsymbol{B}_{\boldsymbol{i}}$ is the time-dependent background counting rate,

$\boldsymbol{B}_{S}$ and $\boldsymbol{B}_{\boldsymbol{O}}$ are the steady state background counting rates for sample and open measurements, respectively, and

$\boldsymbol{K}_{S}$ and $\boldsymbol{K}_{\boldsymbol{O}}$ are the normalization factors for the sample and open measurements.

The normalization factor, $\boldsymbol{K}_{\boldsymbol{O}}$, was determined by forcing the smooth analytical background function through a particular notch-extrapolated background point. The $\mathrm{Nb}$ sample normalization factor, $\boldsymbol{K}_{\boldsymbol{S}}$, was obtained by forcing the smooth analytical background function through the niobium data at that point.

\section{Results}

Resonance parameters for niobium were extracted from a combined fit of capture and transmission data using the computer code SAMMY. A key factor in obtaining good fits to the data was accounting for resolution broadening. Resolution broadening refers to the combined effects of linac burst width, the decay time in the neutron-producing target, the time delay in the moderator, the TOF channel width, and the effect of the detector system. Burst width and channel width are handled explicitly. All the other effects are incorporated into a single resolution function. SAMMY contains a special resolution function for transmission measurements, which was tailored to the RPI experimental apparatus and implemented in SAMMY $^{1}$. To obtain the best possible fits to the data, a new set of parameters to use in the RPI transmission resolution function was derived for this experiment by fitting ${ }^{238} \mathrm{U}$ transmission data with SAMMY. To estimate the error associated with this resolution function, a sensitivity analysis was performed using a method developed at $\mathrm{RPI}^{8}$. The RPI resolution function is essentially a chi-squared function plus an exponential function. It was determined that the resolution function is most sensitive to the $\Lambda$ and $\tau$ parameters of the RPI resolution function. The $\Lambda$ parameter gives the amplitude of the exponential in time and the $\tau$ parameter gives the offset in time. The first step in the sensitivity analysis was to determine the necessary perturbation (plus and minus) to produce a $10 \%$ change in the reduced chi-square of a fit to the uranium data. The next step was to run combined fits of niobium capture and transmission with one of the resolution function parameters perturbed by the amount determined in the first step. Since there were four perturbations $\left(\Lambda^{+}, \Lambda_{-}^{-}, \tau+\right.$, and $\tau$-) four different fits were done. The final 
step was to compare the results of these fits with the final results for niobium resonance parameters, calculating the effects of the $10 \%$ change in $\Lambda$ and $\tau$. These effects were added in quadrature to give the estimated errors associated with the transmission resolution function.

Table II compares the results of this experiment with the resonance parameters listed in ENDF/B-VI. The resonances are grouped into p-wave and s-wave sets. A resonance was added at $192.41 \mathrm{eV}$. This resonance was identified in 1966 by Lopez et al. ${ }^{9}$, but the only resonance parameter assigned was $\Gamma \gamma$. The resonance was also observed in the present experiment, and it was determined that adding the resonance to the niobium set significantly improved the SAMMY fits. The resonance was assigned as an s-wave with $\mathrm{j}=4$ since that assignment resulted in the best SAMMY fit of the data (multiplicity data were inconclusive). Perhaps the greatest change from the ENDF/B-VI values is in the $378.47 \mathrm{eV}$ resonance where the $\Gamma \gamma$ result is $92.3 \mathrm{meV}$ (compared to $174.00 \mathrm{meV}$ for ENDF) and the $\Gamma_{\mathrm{n}}$ result is $114.9 \mathrm{meV}$ (compared to $97.27 \mathrm{meV}$ for ENDF). When only the transmission data from this experiment are fitted, the parameters for this resonance are similar to ENDF. However, including capture results and performing a combined fit of capture and transmission gives the result shown in Table II. It should be noted that the only other experiment (other than the present experiment) to include capture measurements (Lopez et al in 1966) produced results much closer to the present experiment (the $\Gamma \gamma$ result was $140 \pm 15 \mathrm{meV}$ and $2 \mathrm{~g} \Gamma_{\mathrm{n}}$ result was $114 \pm 6 \mathrm{meV}$ for the $378.47 \mathrm{eV}$ resonance).

Table II lists errors from several different sources. The errors listed with the values for $\Gamma \gamma$ and $\Gamma_{\mathrm{n}}$ are the one sigma statistical errors from the combined SAMMY analysis of capture and transmission. The second column of errors for $\Gamma \gamma$ and $\Gamma_{\mathrm{n}}$ gives the estimate of error from the sensitivity analysis of the RPI transmission resolution function (previously discussed in this section). A third column of errors for $\Gamma \gamma$ and $\Gamma_{\mathrm{n}}$ represents the error associated with normalizing the capture data. A quadrature sum of the three errors (statistical, resolution function, and capture normalization) is shown in a fourth column. Energy errors are based on the errors from the SAMMY analysis of capture and transmission, rounded up to the nearest one-hundredth of a percent. An additional analysis of the error associated with the resolution function is given in the appendix.

Figures 1 and Figure 2 give an overview of niobium capture and transmission data over the range of energies analyzed. The fit from a six-sample combined SAMMY analysis is plotted along with the data. Figure 3 and Figure 4 show transmission and capture data with fits for the $119 \mathrm{eV}$ resonance (the resonance that was used to normalize the capture data). All of the samples in the six-sample combined SAMMY analysis are plotted. For comparison, a fit using the ENDF/B-VI resonance parameters is shown for the thickest transmission and capture samples. Figure 5 and Figure 6 show the transmission and capture data for the $194 \mathrm{eV}$ resonance. It should be noted that the energy from the six-sample combined SAMMY analysis $(194.25 \mathrm{eV})$ is significantly different from the ENDF/B-VI value $(193.00 \mathrm{eV})$. Figure 7 and Figure 8 show the transmission and capture data for the $378 \mathrm{eV}$ resonance. In this case, the ENDF/B-VI parameters fit well for the transmission data, but not for the capture data. The value 
of doing a combined SAMMY analysis of capture and transmission, is that the resulting parameters fit both capture and transmission data.

\section{Summary and conclusion}

Neutron capture and transmission measurements of niobium were performed at the RPI linac using the time-of-flight method. The multi-level R-matrix Bayesian code SAMMY was used to perform a combined analysis of capture and transmission data. Resonance parameters were determined for all resonances up to $500 \mathrm{eV}$. Since accounting for resolution broadening was a key factor in obtaining good fits to the data, a new set of parameters to use in the RPI transmission resolution function was derived for this experiment by fitting ${ }^{238} \mathrm{U}$ transmission data with SAMMY. The results show several differences from the ENDF/B-VI values. The greatest change from the ENDF/B-VI values is in the $378.47 \mathrm{eV}$ resonance, where the $\Gamma \gamma$ result is 92.3 $\mathrm{meV}$ (compared to $174.00 \mathrm{meV}$ for ENDF) and the $\Gamma_{\mathrm{n}}$ result is $114.9 \mathrm{meV}$ (compared to 97.27 $\mathrm{meV}$ for ENDF). This change is primarily due to the combined analysis, which includes both capture and transmission data. Also, the energy for the $193.00 \mathrm{eV}$ resonance listed in ENDF/BVI is found to be $194.25 \mathrm{eV}$. Finally, parameters were assigned for a new resonance at 192.41 $\mathrm{eV}$. 


\section{Table II}

\section{Comparison of ENDF/B-VI Resonance Parameters with Present Experiment and Presentation of Errors}

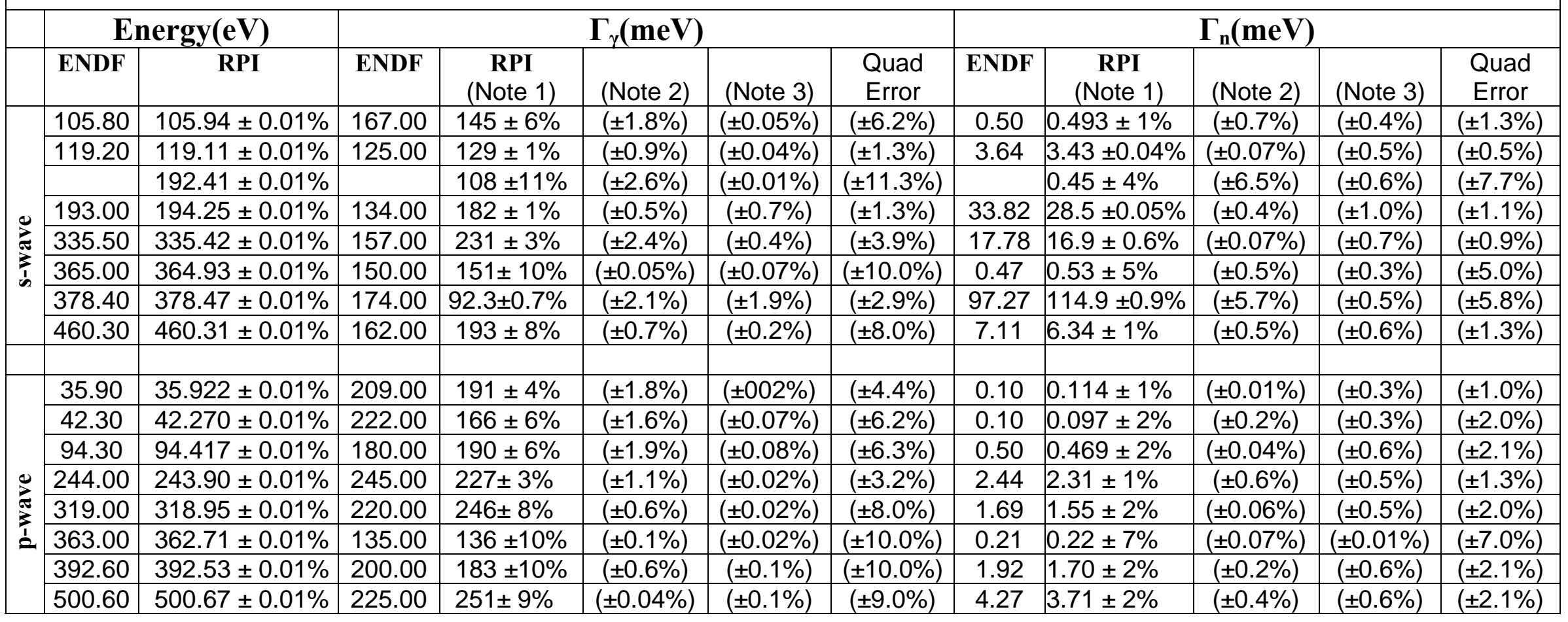

Note 1 -- Both value and error are from combined SAMMY analysis of capture and transmission

Note 2 -- Uncertainty associated with transmission resolution function. (The capture resolution function uncertainty is negligible)

Note 3 - Error associated with capture normalization 
FIGURE 1 -- NIOBIUM CAPTURE WITH FIT. PARAMETERS ARE FROM A SIX-SAMPLE COMBINED FIT.

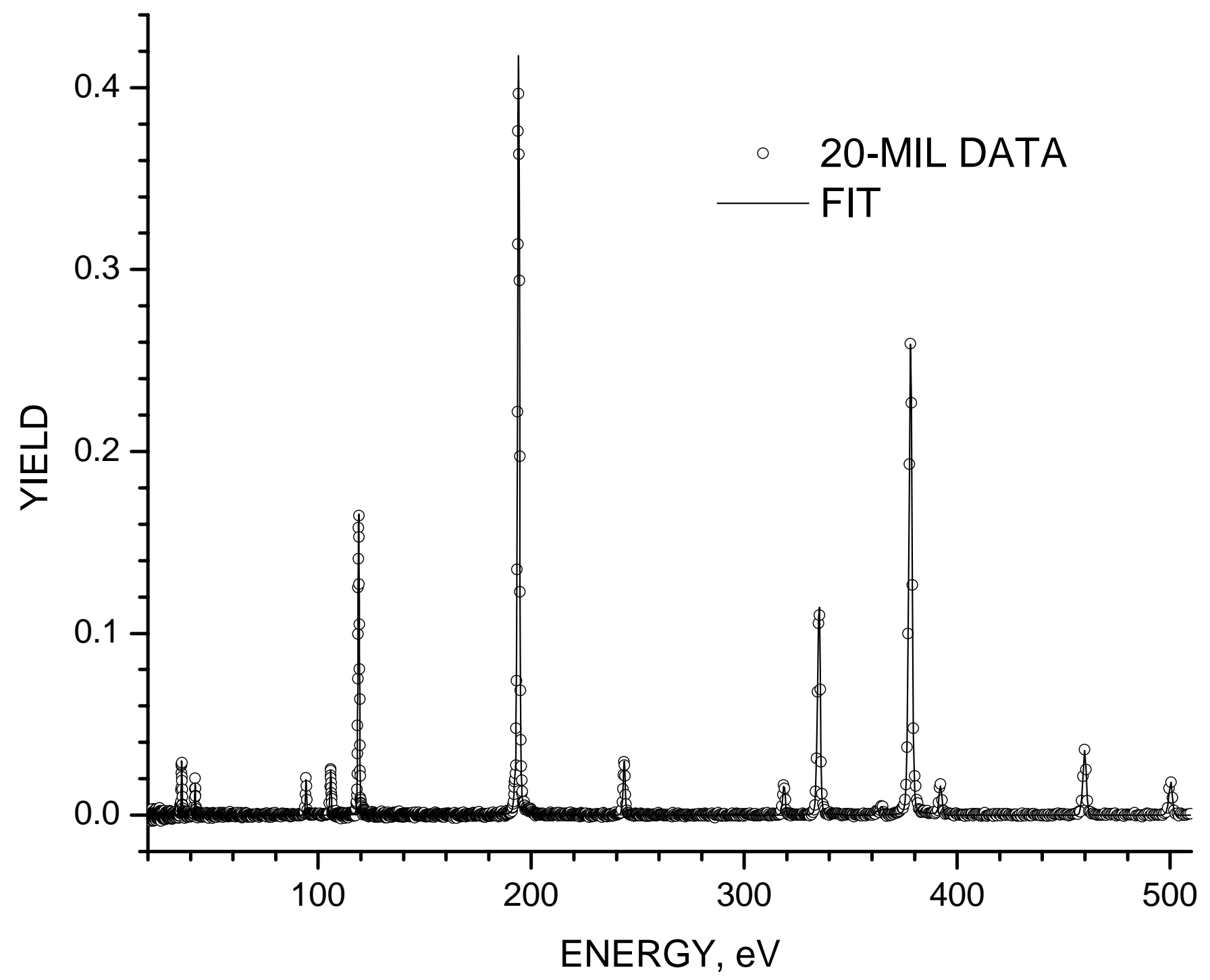


FIGURE 2 -- NIOBIUM TRANSMISSION WITH FIT. PARAMETERS ARE FROM A SIX-SAMPLE COMBINED FIT.

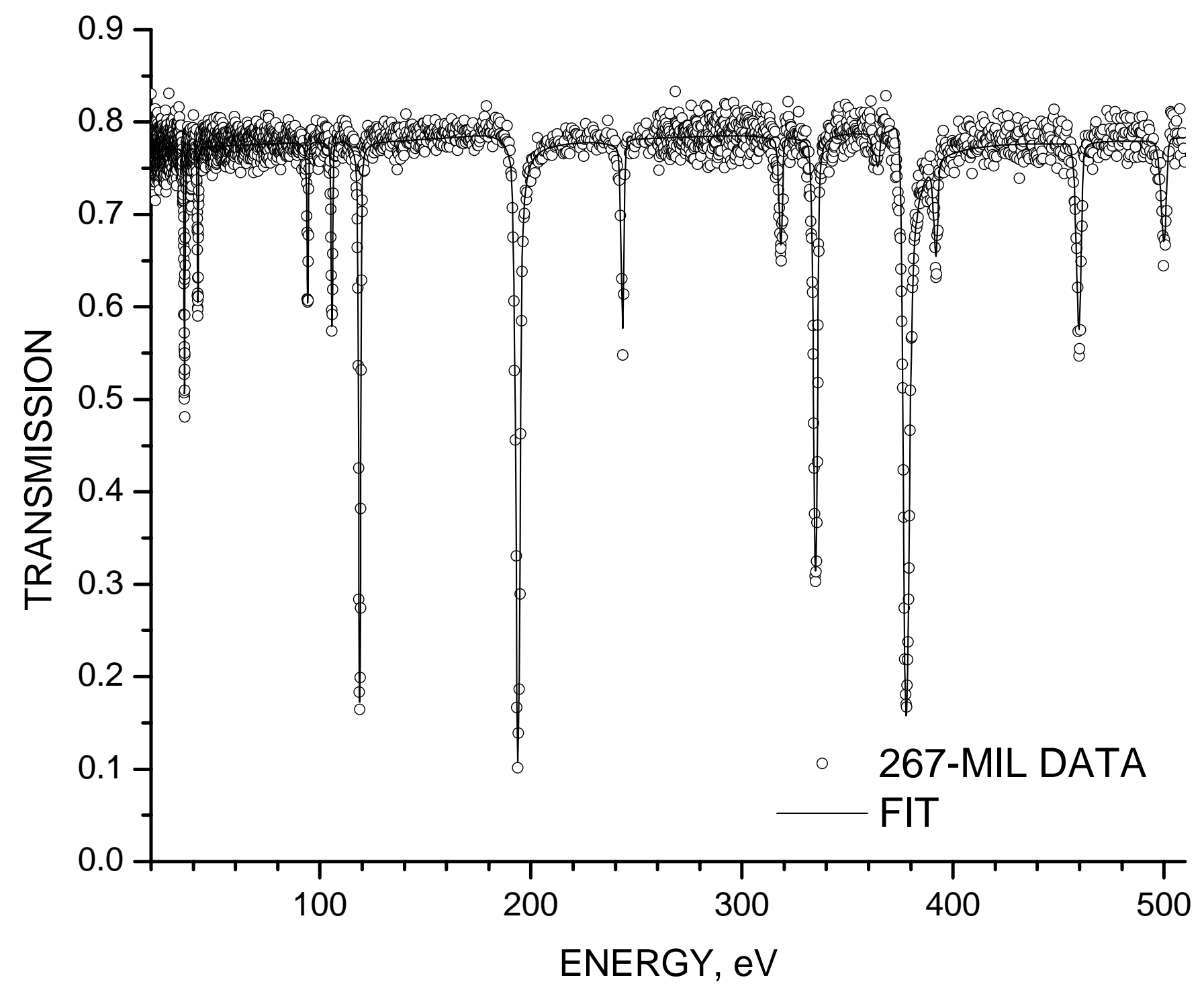


FIGURE 3 -- NIOBIUM TRANSMISSION FOR 135 AND 267-MIL SAMPLES WITH SAMMY FITS. ENDF IS FIT FOR 267-MIL SAMPLE.

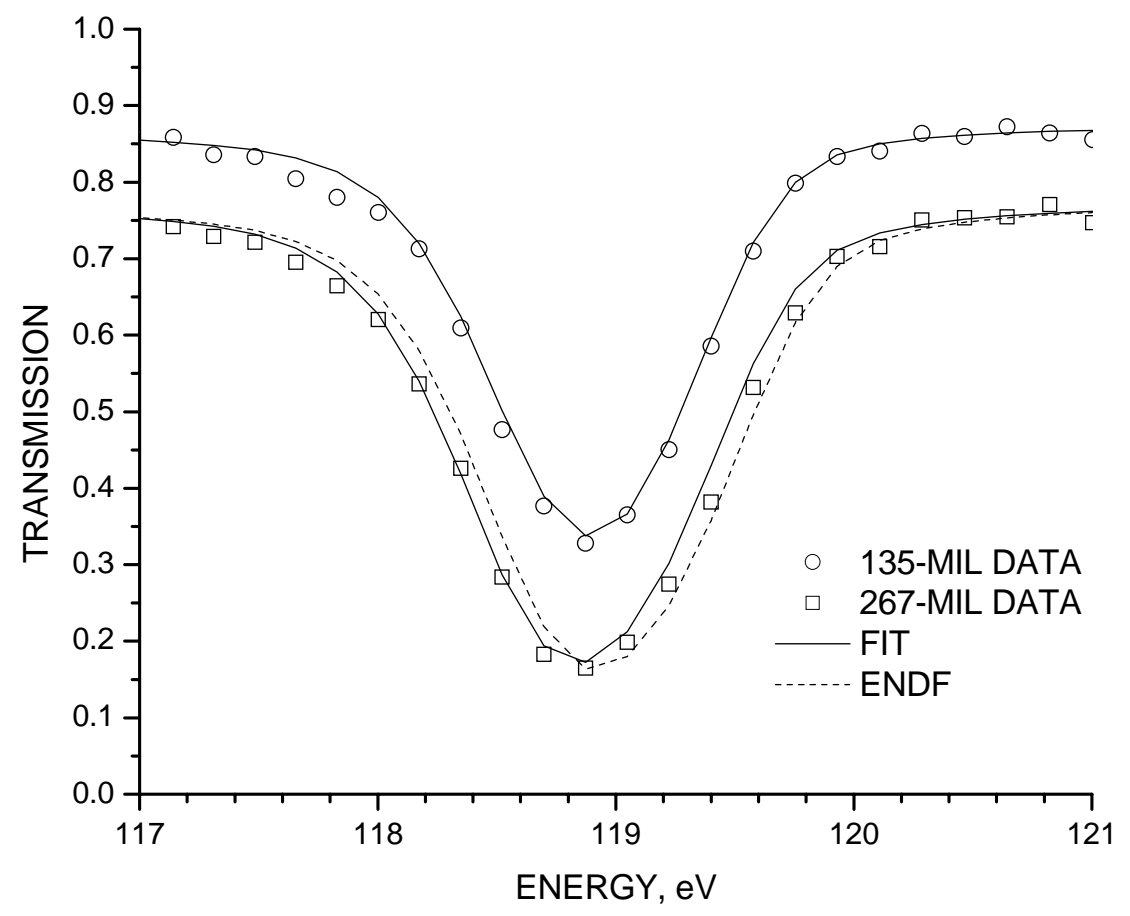

FIGURE 4 -- NIOBIUM CAPTURE FOR 2,5,10,AND 20-MIL SAMPLES WITH SAMMY FITS. ENDF IS FIT FOR 20-MIL SAMPLE.

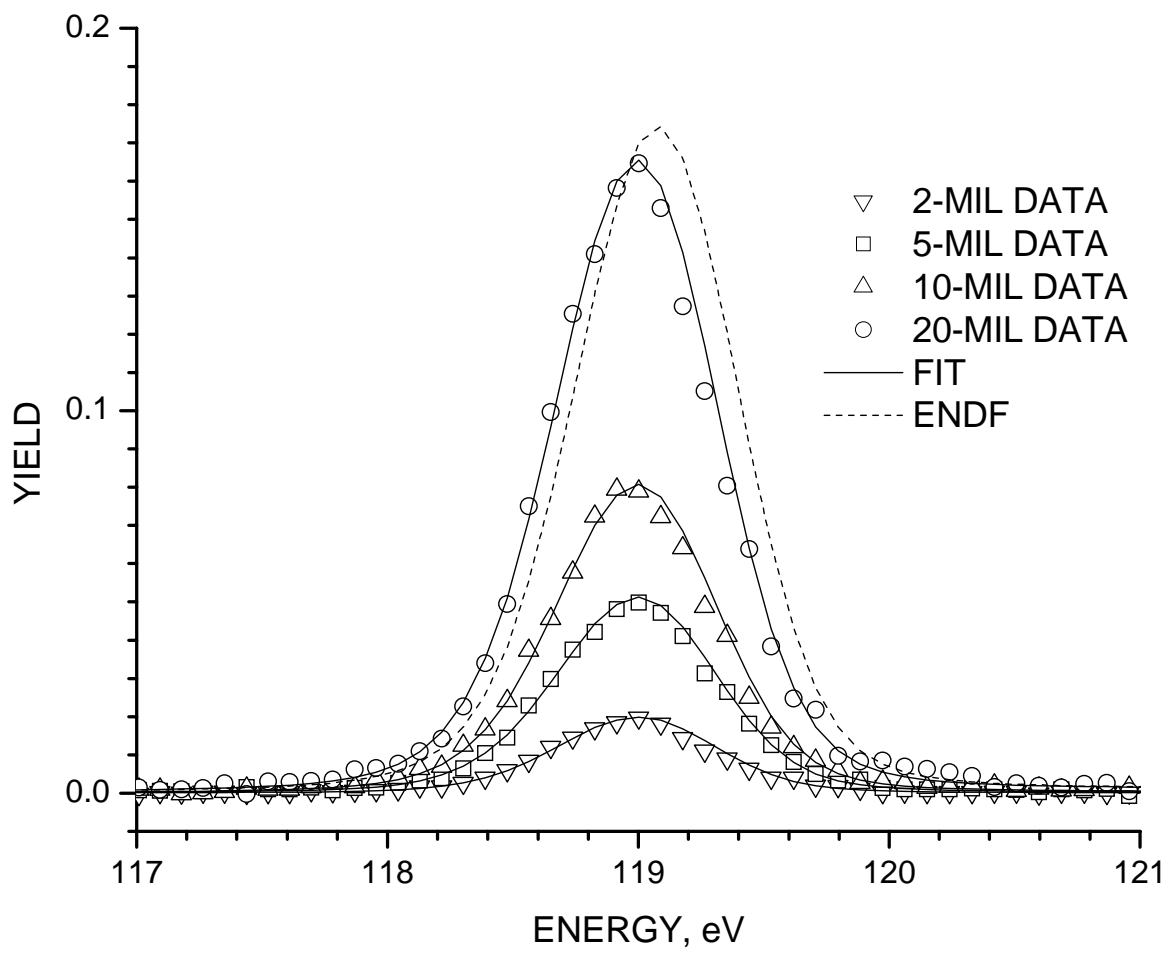


FIGURE 5 -- NIOBIUM TRANSMISSION FOR 135 AND 267-MIL SAMPLES WITH SAMMY FITS. ENDF IS FIT FOR 267-MIL SAMPLE.

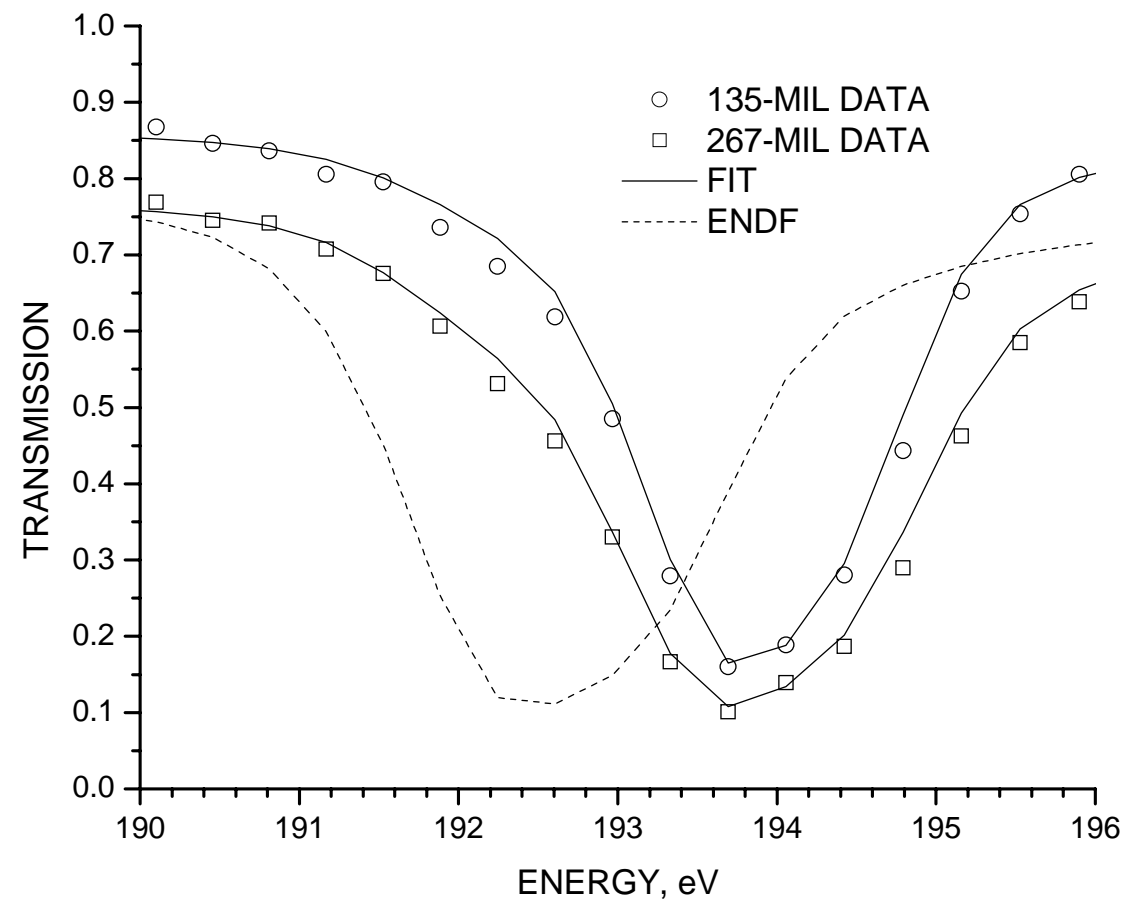

FIGURE 6 -- NIOBIUM CAPTURE FOR 2,5,10,AND 20-MIL SAMPLES WITH SAMMY FITS. ENDF IS FIT FOR 20-MIL SAMPLE.

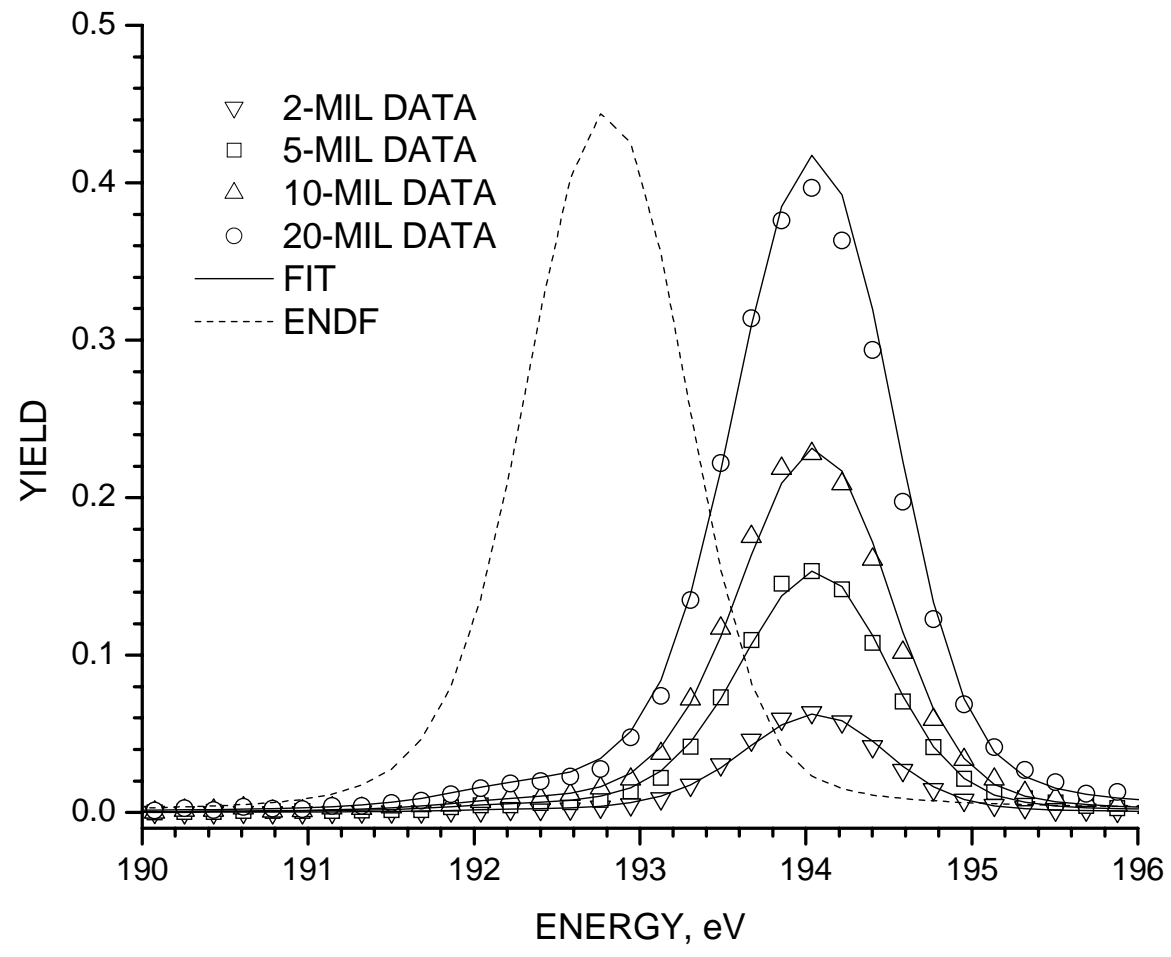




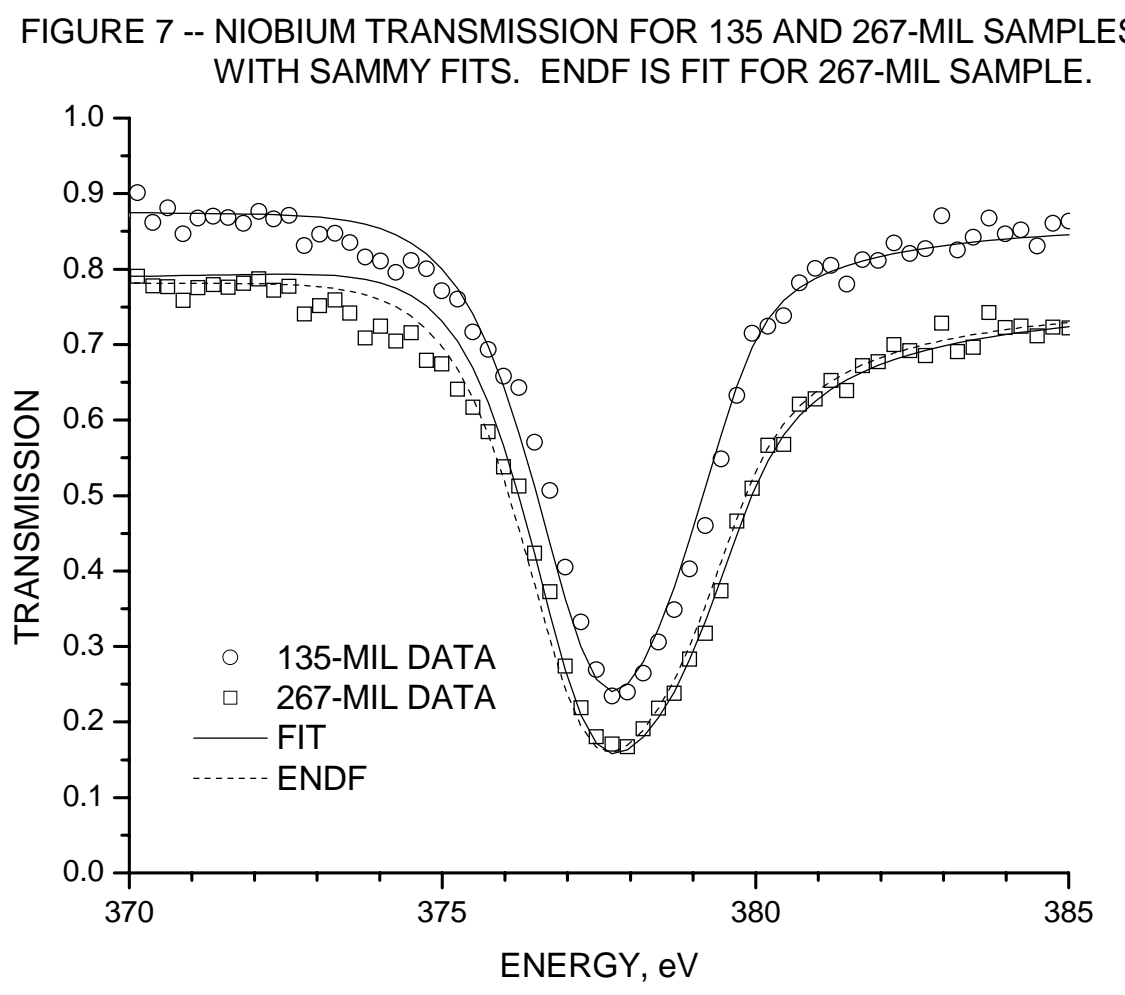

FIGURE 8 -- NIOBIUM CAPTURE FOR 2,5,10,AND 20-MIL SAMPLES WITH SAMMY FITS. ENDF IS FIT FOR 20-MIL SAMPLE.

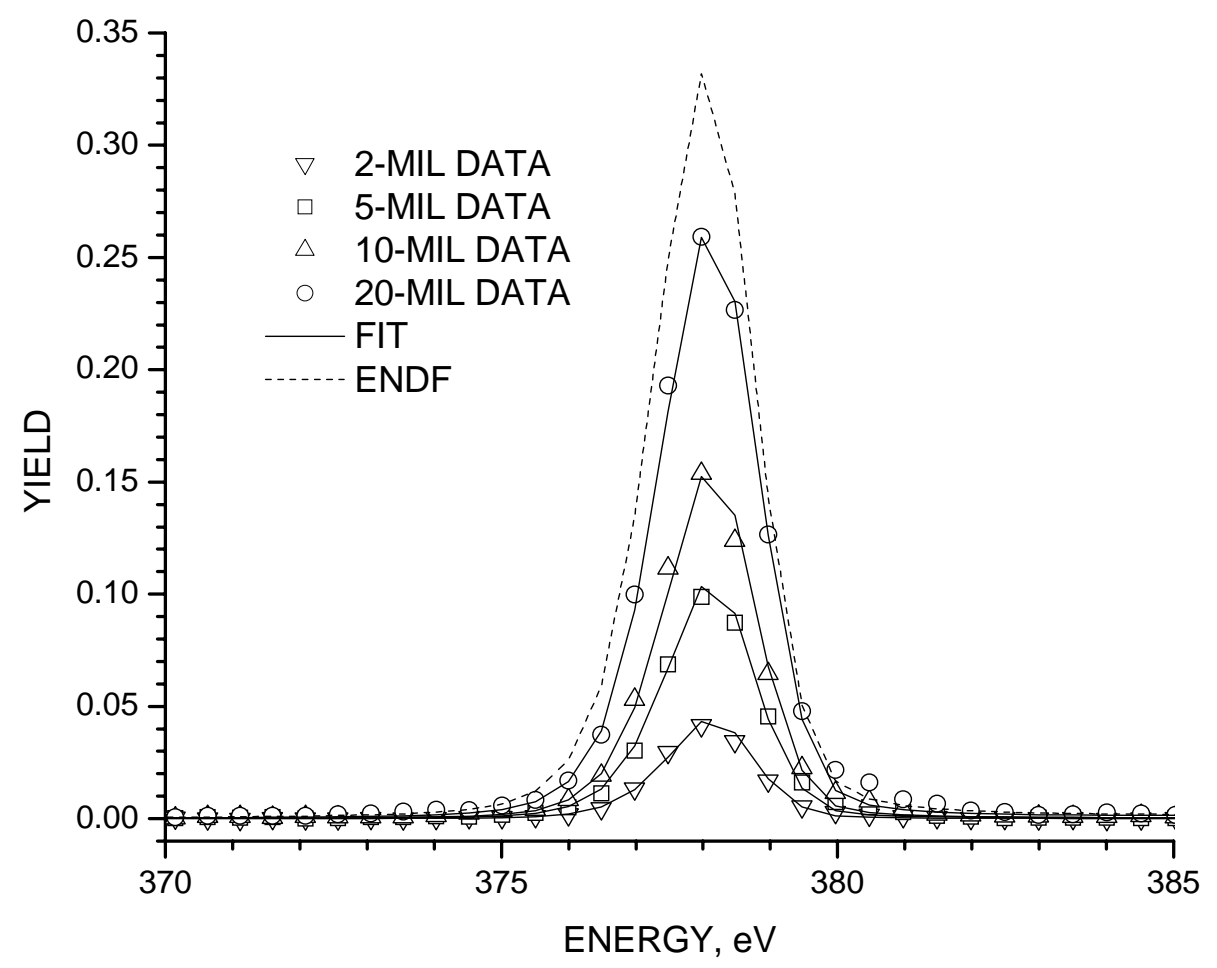




\section{References}

${ }^{1}$ M.E. OVERBERG, B.E. MORETTI, R.E. SLOVACEK, R.C. BLOCK, "Photoneutron Target Development for the RPI Linear Accelerator", Nuclear Instruments and Methods Phys. Res. A, 438, 253 (1999).

${ }^{2}$ R.C. BLOCK, P.J. MARANO, N.J. DRINDAK, F. FEINER, K.W. SEEMANN and R.E. SLOVACEK, "A Multiplicity Detector for Accurate Low-Energy Neutron Capture Measurements," Proc. Int. Conf. Nuclear Data for Science and Technology, Mito, Japan, May 30-June 3, 1988; p.383.

${ }^{3}$ D.P.BARRY, "Neodymium Neutron Transmission and Capture Measurements and Development of a New Transmission Detector", Doctoral Thesis, Rensselaer Polytechnic Institute, 2003.

${ }^{4}$ N.M. LARSON, “Updated Users' Guide for SAMMY: Multilevel R-Matrix Fits to Neutron Data Using Bayes’ Equations” ORNL/TM-9179/R4, Lockheed Martin Energy Research Corp., Oak Ridge National Laboratory, (1998).

${ }^{5}$ R.E. SLOVACEK, R.C. BLOCK, Y. DANON, C.J. WERNER, G.U. YOUK, J.A. BURKE, N.J. DRINDAK, F. FEINER, J.A. HELM, and K.W. SEEMANN, "Neutron Cross Section Measurements at the Rensselaer LINAC," Proc. Topl. Mtg. Advances in Reactor Physics, Knoxville, Tennessee, April 11-15, 1994, Vol. II, p.193, American Nuclear Society (1994)

${ }^{6}$ R.C. BLOCK, Y. DANON, C.J. WERNER, G.U. YOUK, J.A. BURKE, N.J. DRINDAK, F. FEINER, J.A. HELM, J.C. SAYRES and K.W. SEEMANN, "Neutron Time-Of Flight Measurements at the Rensselaer LINAC," Proc. Int. Conf. Nuclear Data for Science and Technology, Gatlinburg, Tennessee, May 9-13, 1994, Vol. 1. p81, American Nuclear Society (1994).

${ }^{7}$ D.B.SYME, "The Black and White-Filter Method for Background Determination in Neutron Time-of-Flight Spectrometry”, Nuclear Instruments and Methods, 198, 357 (1982).

${ }^{8}$ M.J.TRBOVICH, "Hafnium Neutron Cross Sections and Resonance Analysis", Doctoral Thesis, Rensselaer Polytechnic Institute, 2003.

9 W.M. LOPEZ, E HADDAD, S.J. FRIESENHAHN, AND F.H.FROHNER, "Niobium Resonance Parameters”, Nuclear Physics, A93, 340-356 (1967). 


\section{Appendix}

An additional analysis of the error associated with the resolution function was performed using a different method. Two independent sets of resolution function parameters for the RPI resolution function were used to do transmission-only fits of the niobium data. Each independent set of resolution function parameters was derived by fitting a different uranium data set with SAMMY. One was derived as part of the data analysis in the present experiment. The other was derived by Trbovich as part of an analysis of hafnium data ${ }^{8}$. The error associated with the resolution function was determined by comparing transmission-only fits of the niobium data over a range of 10-200 eV. The range was restricted because of limitations in the data used by Trbovich. However, the two sets of resolution function parameters (both done at the RPI linac) are judged to be of equivalent quality over this energy range. Table III gives the results of this comparison.

\section{Table III}

\section{Comparison of ENDF/B-VI Resonance Parameters with Present Experiment and comparison of errors from two sets of resolution function parameters}

\begin{tabular}{|c|c|c|c|c|c|c|c|c|}
\hline & \multicolumn{2}{|c|}{$\operatorname{Energy}(e V)$} & \multicolumn{3}{|c|}{$\Gamma_{\gamma}(\mathrm{meV})$} & \multicolumn{3}{|c|}{$\Gamma_{\mathrm{n}}(\mathrm{meV})$} \\
\hline & ENDF & RPI (Note 1) & ENDF & RPI(Note1) & (Note2) & ENDF & RPI(Note1) & (Note2) \\
\hline \multirow{4}{*}{$\sum_{i}^{\infty}$} & 105.80 & $105.943 \pm 0.005 \%$ & 167.00 & $145 \pm 6 \%$ & $\pm 11 \%$ & 0.50 & $0.493 \pm 1 \%$ & $\pm 3 \%$ \\
\hline & 119.20 & $119.112 \pm 0.0008 \%$ & 125.00 & $129 \pm 1 \%$ & $\pm 17 \%$ & 3.64 & $3.43 \pm 0.04 \%$ & $\pm 3 \%$ \\
\hline & & $192.41 \pm 0.01 \%$ & & $108 \pm 11 \%$ & $\pm 3 \%$ & & $0.45 \pm 4 \%$ & $\pm 19 \%$ \\
\hline & 193.00 & $194.249 \pm 0.0007 \%$ & 134.00 & $182 \pm 1 \%$ & $\pm 6 \%$ & 33.82 & $28.5 \pm 0.05 \%$ & $\pm 4 \%$ \\
\hline \multirow{3}{*}{ 竞 } & 35.90 & $35.922 \pm 0.007 \%$ & 209.00 & $191 \pm 4 \%$ & $\pm 3 \%$ & 0.10 & $0.114 \pm 1 \%$ & \pm 0.4 \\
\hline & 42.30 & $42.270 \pm 0.01 \%$ & 222.00 & $166 \pm 6 \%$ & $\pm 3 \%$ & 0.10 & $0.097 \pm 2 \%$ & \pm 0.6 \\
\hline & 94.30 & $94.417 \pm 0.006 \%$ & 180.00 & $190 \pm 6 \%$ & $\pm 8 \%$ & 0.50 & $0.469 \pm 2 \%$ & $\pm 1 \%$ \\
\hline
\end{tabular}

Note 1 -- Both value and error are from combined SAMMY analysis of capture and transmission

Note 2 - Error associated with the transmission resolution function, obtained by comparing two transmission-only fits of the niobium data, each using an independent set of resolution function parameters. 